\title{
Reflection and noise in the low spectral state of GX 339-4
}

\author{
M. Revnivtsev ${ }^{1,2}$, M. Gilfanov ${ }^{1,2}$, and E. Churazov ${ }^{1,2}$ \\ 1 Space Research Institute, Russian Academy of Sciences, Profsoyuznaya 84/32, 117810 Moscow, Russia \\ 2 Max-Planck-Institute für Astrophysik, Karl-Schwarzschild-Str. 1, 85740 Garching bei München, Germany
}

Received 6 February 2001 / Accepted 4 October 2001

\begin{abstract}
We analyze RXTE/PCA observations of GX 339-4 in the low spectral state from 1996-1997 and show that the pattern of its spectral and temporal variability is nearly identical to that of Cyg X-1. In particular, a tight correlation exists between the QPO centroid frequency and the spectral parameters. An increase of the QPO centroid frequency is accompanied with an increase of the amplitude of the reflected component and a steepening the slope of the underlying power law. Fourier frequency resolved spectral analysis showed, that the variability of the reflected component at frequencies higher than $\sim 1-10 \mathrm{~Hz}$ is suppressed in comparison with that of the primary emission.
\end{abstract}

Key words. accretion, accretion disks - black hole physics - stars: binaries: general - stars: individual: GX 339-4 X-rays: general - X-rays: stars

\section{Introduction}

It was found recently for a large sample of Seyfert AGNs and several observations of the Galactic X-ray binaries that the amplitude of the reflected component is generally correlated with the slope of the primary power law emission (Zdziarski et al. 1999). Based on the numerous RXTE/PCA observations of Cyg X-1 Gilfanov et al. (1999) (hereafter Paper I) showed that this correlation is strong for multiple observations of this source and that the spectral parameters are also tightly correlated with the characteristic noise frequency. In particular an increase of the QPO centroid frequency is accompanied with a steepening of the slope of the Comptonized radiation and an increase of the amplitude of the reflected component. Studying fast variability of the reflected emission Revnivtsev et al. (1999) showed that its amplitude is suppressed with respect to that of the primary emission at the frequencies higher than $\sim 1-10 \mathrm{~Hz}$.

GX 339-4 is a bright and well studied X-ray binary. It is usually classified as a black hole candidate and in many aspects is very similar to Cyg X-1 (see e.g. Tanaka \& Lewin 1995; Trudolyubov et al. 1998; Zdziarski et al. 1998; Wilms et al. 1999; Nowak et al. 1999). The investigations of the connections between the spectral and timing properties of Cyg X-1 (e.g. Gilfanov et al. 1999)

Send offprint requests to: M. Revnivtsev,

e-mail: revnivtsev@hea.iki.rssi.ru and GX 339-4 (e.g. Ueda et al. 1994) indicate that these sources could be similar from this point of view also. In this paper we expand the analysis of correlations between spectral and temporal characteristics of the X-ray emission of GX 339-4 in the low spectral state using the Rossi X-ray Timing Explorer data and show that this source demonstrates the same behavior that was previously observed from Cyg X-1.

\section{Observations, data reduction and analysis}

We used the publicly available data of GX 339-4 observations with RXTE/PCA from 1996-1997 performed during the low spectral state of the source. Our sample includes 23 observations from the proposals 10068, 20056 , 20181 and 20183 with a total exposure time of $\sim 130 \mathrm{ksec}$ (Table 1). Only observations from the proposal P20183 had sufficient energy and timing resolution to perform Fourier frequency resolved spectral analysis. Therefore the frequency resolved analysis was carried out only for $\sim 61 \mathrm{ksec}$ of the data.

The data screening was performed following the RXTE GOF recommendations: offset angle $<0.02^{\circ}$, Earth elevation angle $>10^{\circ}$, electron contamination value (the "electron ratio") for any of PCUs $<0.1$. The data from all PCUs were used for the analysis. The energy spectra were extracted from the PCA mode "Standard 2" (128 channels, $16 \mathrm{~s}$ time resolution) and averaged over each observation. Fourier frequency resolved spectral analysis used 


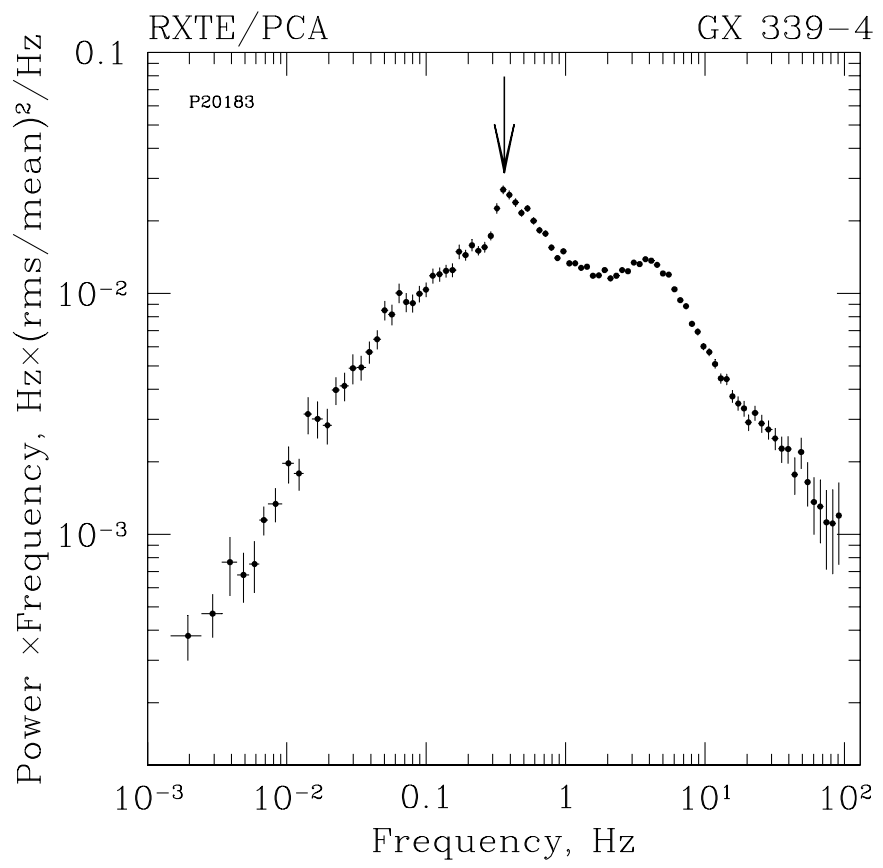

Fig. 1. Typical power density spectrum of GX 339-4 in the low spectral state. The QPO peak at the frequency of $\sim 0.35 \mathrm{~Hz}$ is shown by the arrow. In order to improve the statistics the power density spectrum was averaged over all observations of the proposal P20183 having somewhat different QPO centroid frequency, resulting in a somewhat broader profile of the QPO peak.

"Good Xenon" data (256 energy channels, $1 \mu$ s time resolution). The response matrixes were built using standard RXTE FTOOLS 4.2 tasks (Jahoda 1999). The background spectra for the conventional spectral analysis were constructed with the help of the "VLE" based model (Stark 1999). The background contribution to the frequency resolved spectra is negligible in the frequency and energy ranges of interest. A uniform systematic uncertainty of $0.5 \%$ was added quadratically to the statistical error in each energy channel. The value of systematic uncertainty was chosen basing on the deviations of the PCA Crab spectra from a power law model (see e.g. Wilms et al. 1999).

The energy spectra were fit in the $3-20 \mathrm{keV}$ energy range with a spectral model identical to that of Paper I. The model consisted of a power law without high energy cutoff with superposed continuum, reflected from the neutral medium (pexrav model in XSPEC, see Magdziarz \& Zdziarski 1995) and an intrinsically narrow emission line at the energy $6.4 \mathrm{keV}$. No ionization effects were taken into account. The binary system inclination angle was fixed at $\theta=45^{\circ}$ (e.g. Zdziarski et al. 1998), the iron abundance at the solar value. In such a model the amplitude of the reflected component is characterized by the reflection scaling factor $R$, which is an approximate measure of the solid angle subtended by the reflector, $R \sim \Omega / 2 \pi$. In the simplest geometry of an isotropic point source above the infinite reflecting plane, the reflection scaling factor $R$ is equal to 1 . In order to approximately account for smearing of the reflection features due to e.g. relativistic and ionization effects the reflected continuum and the fluorescent line were convolved with a Gaussian. Its width was a free parameter of the fit. The uncertainties in Table 1 represents $1 \sigma$ confidence intervals for the model parameters. The error bars on the values of equivalent width of the line were calculated by the propagation of errors from line flux value.

The power spectra of GX 339-4 in the low spectral state feature a prominent QPO peak which frequency varies typically between $\sim 0.1$ and $\sim 0.5 \mathrm{~Hz}$ (Fig. 1 ). We therefore used its centroid to parameterize the characteristic noise frequency. The power spectra were approximated with a model consisting of two band limited noise components (Lorentzians, centered at zero frequency) and the comparably narrow Lorentzian profile (QPO peak).

The Fourier frequency resolved spectra were obtained following the prescription of Revnivtsev et al. (1999) and were approximated with the same model as averaged spectra except that the width of the Gaussian used to model the smearing of the reflection features was fixed at the value of $0.7 \mathrm{keV}$.

\section{Results and their uncertainties}

The results of the energy and power spectra approximation are presented in Table 1 and Fig. 2. As can be seen from Fig. 2 the main temporal and spectral parameters - characteristic noise frequency, slope of the underlying power power law and amplitude of the reflected component - change in a correlated way. A steepening of the spectrum is accompanied with an increase of the reflection amplitude and an increase of the QPO centroid frequency. Such a behavior is very similar to that found in Paper I for $\mathrm{Cyg} \mathrm{X}-1$.

The spectral model is obviously oversimplified and does not include several important effects such as ionization of the reflecting media, deviations of the primary emission spectrum from the power law, exact shape of the relativistic smearing of the reflection features etc. These effects might affect the best fit parameters and could lead to appearance of artificial correlations between them. Particulary sensitive to the choice of the spectral model is the reflection scaling factor $R \sim \Omega / 2 \pi$. As is well known there is some degeneracy between the amplitude of reflection $R$ and the photon index $\Gamma$ of the underlying power law determined from the spectral fits, especially if the spectral fitting was done in a limited energy range (e.g. Zdziarski et al. 1999). This degeneracy might result in a slight positive correlation between the best fit values of $R$ and $\Gamma$ which is in part due to statistical noise and, in part, due to inadequate choice of the spectral model. The statistical part of this degeneracy is illustrated in Fig. 2 by a 2-dimensional confidence contour for one of the points in the $R-\Gamma$ plane. As can be easily seen from Fig. 2 it 


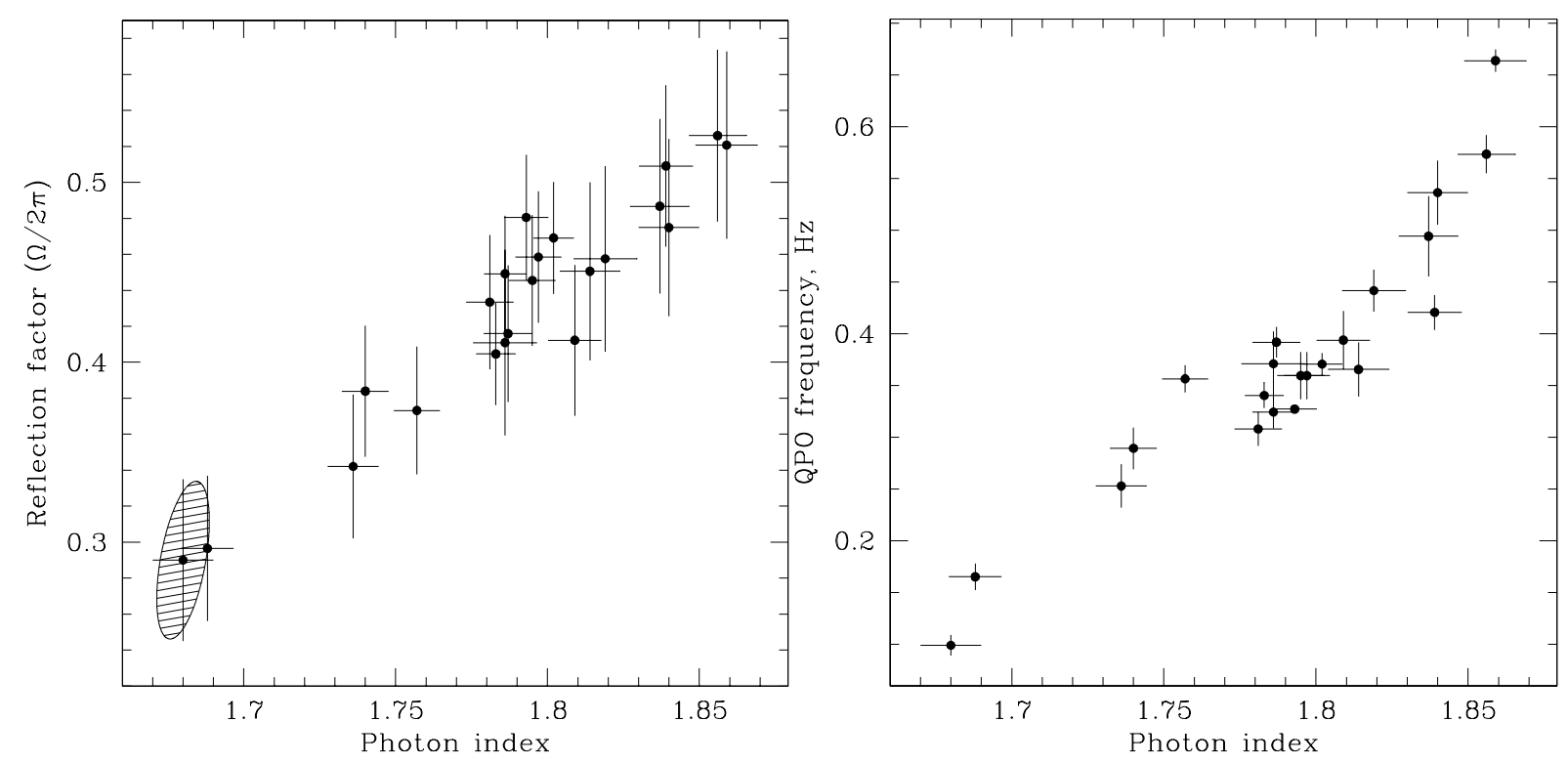

Fig. 2. The dependences of reflection scaling factor and the QPO frequency on the slope of the primary power law. The filled oval shaped region on the left panel shows a typical 1- $\sigma$ confidence contour.

Table 1. The list of observations and parameters of the energy and power density spectra approximation.

\begin{tabular}{|c|c|c|c|c|c|c|c|c|c|}
\hline Obs. ID & Date & Time, UT & Exp. $^{a}$ & $\bar{\Gamma}$ & $R \sim \Omega / 2 \pi$ & $E W, \mathrm{eV}$ & $\overline{\sigma^{b}, \mathrm{keV}}$ & $f_{\mathrm{QPO}}, \mathrm{Hz}$ & $\chi^{2}\left(40_{\text {d.o.f. }}\right)$ \\
\hline 10068-05-01-00 & $17 / 10 / 96$ & $02: 12-05: 07$ & 5550 & $1.68 \pm 0.01$ & $0.29 \pm 0.04$ & $143 \pm 30$ & $0.64 \pm 0.09$ & $0.09 \pm 0.01$ & 26.5 \\
\hline $10068-05-02-00$ & $29 / 10 / 96$ & $22: 33-00: 45$ & 5530 & $1.69 \pm 0.01$ & $0.30 \pm 0.04$ & $120 \pm 30$ & $0.62 \pm 0.14$ & $1 \pm 0.01$ & 37.6 \\
\hline 20056-01-01-00 & $05 / 04 / 97$ & $08: 36-09: 15$ & 2118 & $1.79 \pm 0.01$ & $0.41 \pm 0.05$ & $173 \pm 40$ & $0.85 \pm 0.11$ & $0.37 \pm 0.03$ & 29.0 \\
\hline 20056-01-02-00 & $10 / 04 / 97$ & $2: 28$ & 2071 & $1.81 \pm 0.01$ & $0.41 \pm 0.04$ & $184 \pm 32$ & \pm 0.08 & 0.03 & 38.7 \\
\hline 20056-01-03-00 & $11 / 04 / 97$ & $13: 25-14: 06$ & 2122 & $1.81 \pm 0.01$ & $0.45 \pm 0.05$ & $202 \pm 38$ & $0.83 \pm 0.09$ & $0.36 \pm 0.03$ & 36.2 \\
\hline 20056-01-04-00 & $13 / 04 / 97$ & $20: 09-20: 50$ & 2282 & $1.82 \pm 0.01$ & $0.46 \pm 0.05$ & $213 \pm 41$ & $0.92 \pm 0.09$ & $0.44 \pm 0.02$ & 32.5 \\
\hline 20056-01-05-00 & $15 / 04 / 97$ & $20: 41-21: 20$ & 2049 & $1.84 \pm 0.01$ & $0.47 \pm 0.05$ & $218 \pm 37$ & $0.89 \pm 0.08$ & \pm 0.03 & 27.9 \\
\hline 20056-01-06-00 & $17 / 04 / 97$ & $23: 25-00: 01$ & 2033 & $1.84 \pm 0.01$ & $0.49 \pm 0.05$ & $225 \pm 37$ & $0.92 \pm 0.07$ & $0.49 \pm 0.04$ & 34.2 \\
\hline$-01-07-00$ & $19 / 04 / 97$ & $22: 2$ & 2045 & & $0.53 \pm$ & 216 & $0.79 \pm 0.07$ & $0.57 \pm 0.02$ & \\
\hline 20056-01-08-00 & $22 / 04 / 97$ & $21: 5$ & 1988 & 1 & $0.52 \pm 0.05$ & $227 \pm 38$ & $0.89 \pm 0.07$ & $0.66 \pm 0.01$ & 7 \\
\hline 20181-01-01-01 & $03 / 02 / 97$ & $15: 56-19: 09$ & 6622 & $1.80 \pm 0.01$ & $0.46 \pm 0.04$ & $191 \pm 30$ & $0.84 \pm 0.07$ & $0.34 \pm 0.02$ & 37.7 \\
\hline 20181-01-01-00 & $03 / 02 / 97$ & $22: 26-01: 17$ & 5452 & $1.79 \pm 0.01$ & $0.45 \pm 0.04$ & $196 \pm 30$ & $0.83 \pm 0.07$ & $0.35 \pm 0.02$ & 40.6 \\
\hline 20181-01-02-00 & $10 / 02 / 97$ & $15: 49-20: 22$ & 10535 & $1.79 \pm 0.01$ & $0.48 \pm 0.03$ & $190 \pm 29$ & $0.86 \pm 0.07$ & $0.32 \pm 0.01$ & 22.8 \\
\hline 20181-01-03-00 & $17 / 02 / 97$ & $18: 28-23: 46$ & 11412 & $1.79 \pm 0.01$ & $0.45 \pm 0.03$ & $176 \pm 26$ & $0.79 \pm 0.07$ & $0.32 \pm 0.01$ & 24.3 \\
\hline 20183-01-01-01 & $08 / 02 / 97$ & $14: 20-20: 25$ & 13702 & $1.80 \pm 0.01$ & $0.47 \pm 0.03$ & $185 \pm 26$ & $0.80 \pm 0.06$ & $0.37 \pm 0.02$ & 34.3 \\
\hline 20183-01-02-00 & $14 / 02 / 97$ & $00: 18-06: 33$ & 9780 & $1.78 \pm 0.01$ & $0.40 \pm 0.03$ & $177 \pm 24$ & $0.75 \pm 0.06$ & $0.34 \pm 0.01$ & 21.4 \\
\hline 20183-01-02-01 & $14 / 02 / 97$ & $14: 20-21: 22$ & 5779 & $1.78 \pm 0.01$ & $0.43 \pm 0.04$ & $182 \pm 30$ & $0.81 \pm 0.08$ & $0.30 \pm 0.02$ & 30.0 \\
\hline 20183-01-03-00 & $22 / 10 / 97$ & $03: 00-05: 52$ & 6556 & $1.84 \pm 0.01$ & $0.51 \pm 0.04$ & $203 \pm 35$ & $0.90 \pm 0.08$ & $0.42 \pm 0.02$ & 38.5 \\
\hline 20183-01-04-00 & $25 / 10 / 97$ & $03: 22-06: 00$ & 5385 & $1.79 \pm 0.01$ & $0.42 \pm 0.04$ & $160 \pm 28$ & $0.72 \pm 0.09$ & $0.39 \pm 0.01$ & 35.8 \\
\hline 20183-01-05-00 & $28 / 10 / 97$ & $18: 08-22: 13$ & 6534 & $1.76 \pm 0.01$ & $0.37 \pm 0.04$ & $151 \pm 27$ & $0.72 \pm 0.09$ & $0.36 \pm 0.01$ & 28.2 \\
\hline 20183-01-06-00 & $31 / 10 / 97$ & $19: 41-22: 10$ & 4621 & $1.74 \pm 0.01$ & $0.34 \pm 0.04$ & $129 \pm 29$ & $0.63 \pm 0.12$ & $0.25 \pm 0.02$ & 44.2 \\
\hline 20183-01-07-00 & $03 / 11 / 97$ & $20: 35-23: 48$ & 7186 & $1.74 \pm 0.01$ & $0.38 \pm 0.04$ & $135 \pm 26$ & $0.67 \pm 0.10$ & $0.29 \pm 0.02$ & 30.7 \\
\hline
\end{tabular}

a Dead time corrected value.

${ }^{b}$ The width of the Gaussian used to model the smearing of the reflection features.

is correctly represented by the error bars assigned to the points.

In order to estimate contribution of the second, systematic part of the $R-\Gamma$ degeneracy we compare two pairs of observations with different and close best fit values of reflection factors $R$. We plot in Fig. 3 the ratios of the count spectra for each pair. As is clearly seen from Fig. 3 the spectrum having larger best fit value of the reflection 


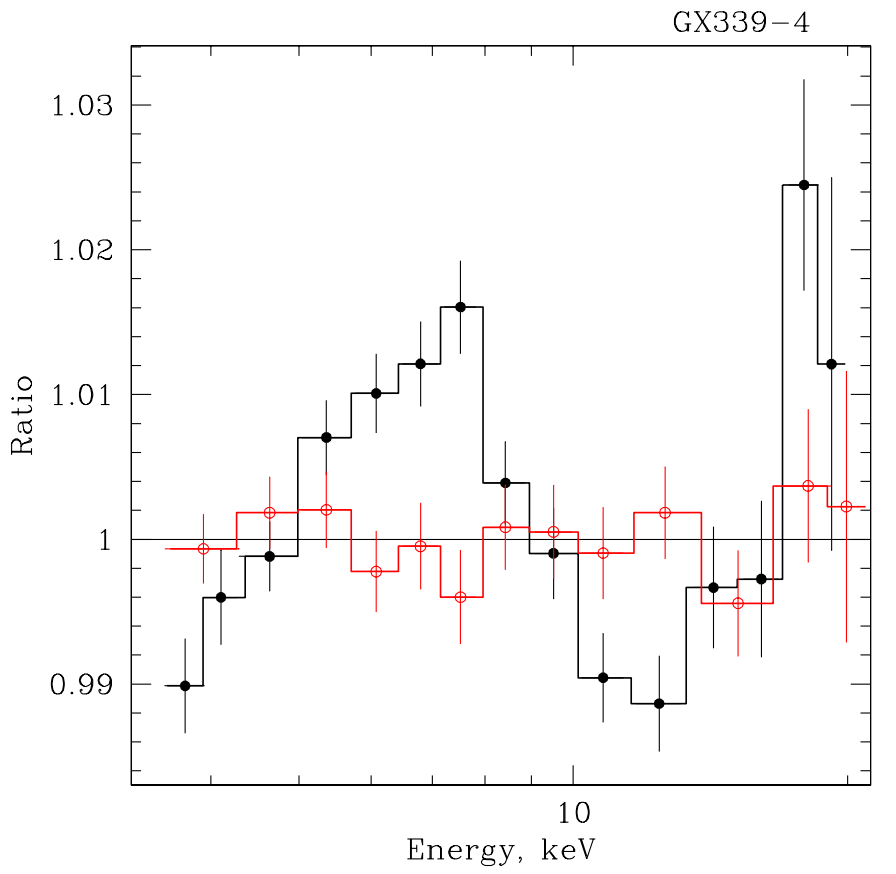

Fig. 3. Ratios of the observed counts spectra with different best fit values of the reflection scaling factor $R$ and photon index $\Gamma$. Solid circles show the ratio of the spectrum averaged over April 13-22, 1997 observations, having large best fit value of the reflection $(R=0.5, \Gamma=1.84)$ to the average spectrum of Oct. 17 and 19, 1996 with the smallest best fit value of reflection and hardest power law photon index $(R=0.3, \Gamma=1.69)$. The ratio was multiplied by $E^{-0.09}$ for clarity. For comparison the open circles show the ratio of the counts spectra of Oct. 25, 1997 and Feb. 14, 1997 having close values of the best fit parameters $(R=0.43, \Gamma=1.78$ and $R=0.42, \Gamma=1.79$ respectively) and separated by the similar period in time as the first ratio.

scaling factor (and the equivalent width of the line) shows more pronounced reflection signatures - the fluorescence line at $\sim 6.4 \mathrm{keV}$ followed by the absorption edge and increase due to the Compton reflected continuum at larger energies. Thus we conclude that although the best fit values of the model parameters might not represent the exact values of the physically important quantities, our spectral model does correctly rank the spectra according to the amplitude of the reflection signatures and the correlations shown in Fig. 2 are not artificial.

The Fourier frequency resolved spectra illustrate the energy dependence of the amplitude of X-ray flux variation at a given time scale. As was stressed out by Revnivtsev et al. (1999) and Gilfanov et al. (2000b) interpretation of the Fourier frequency resolved spectra in general is not straightforward and requires certain a priori assumptions to be made. One of the areas where it can be efficiently used and which at present can not be accessed by the conventional spectroscopy is studying the fast variability of the reflected emission. Indeed, variation of the parameters in the Comptonization region, for instance, lead to variations of the spectral shape of the Comptonized radiation

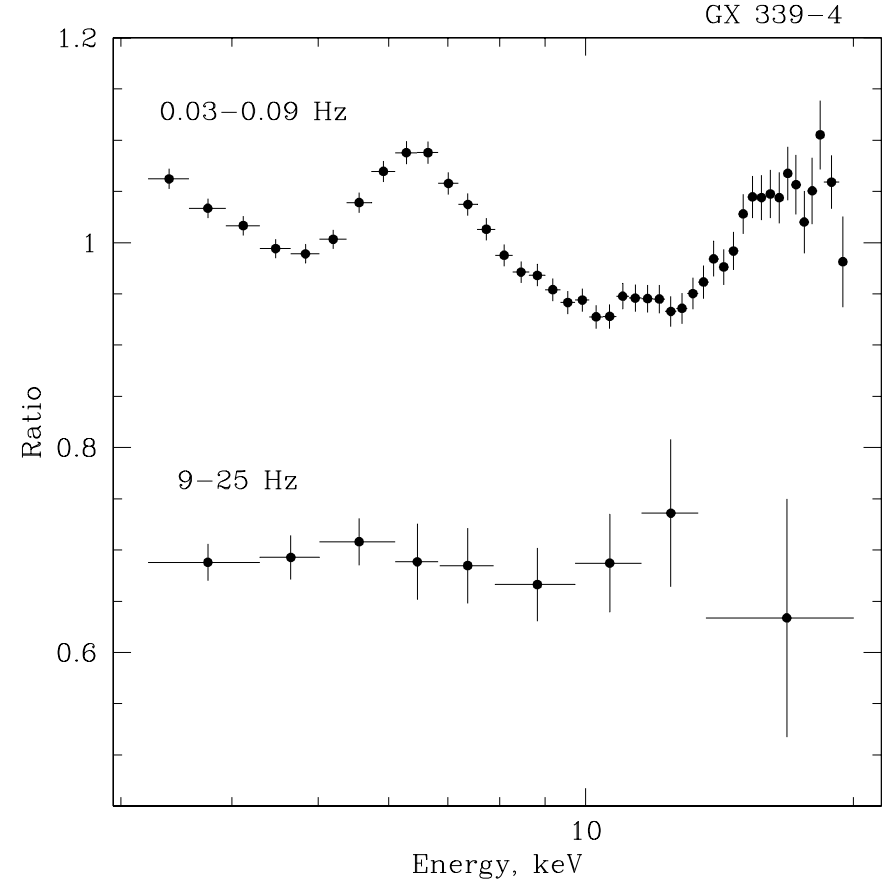

Fig. 4. The ratio of the frequency resolved spectra to a power law model with photon index of $\alpha=1.8$. The $9-25 \mathrm{~Hz}$ spectrum was multiplied by 0.7 for clarity.

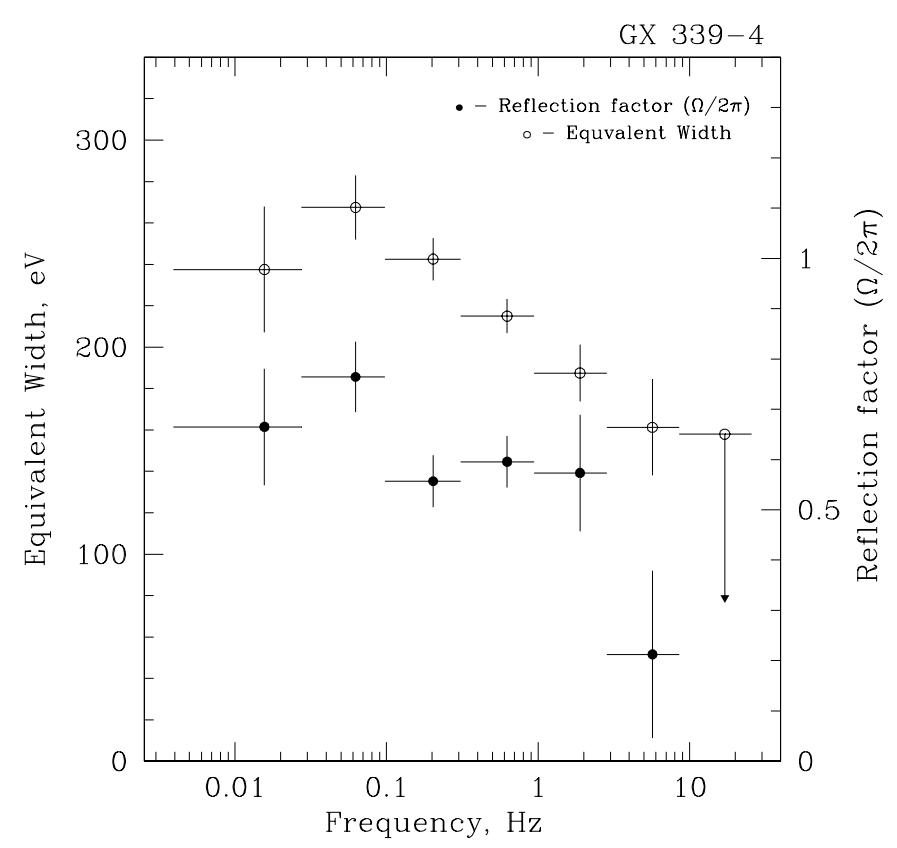

Fig. 5. The Fourier frequency dependence of the equivalent width of the fluorescent line and the reflection scaling factor $R$. The upper limit on the reflection scaling factor in the $9-25 \mathrm{~Hz}$ frequency range equals to 1.5 and not shown in the graph.

which would be imprinted in the Fourier frequency resolved spectra. Their shape, however, might differ significantly from any of the Comptonized spectra they resulted from and no easily interpretable results could be obtained via the conventional spectral fits. The shape of the reflection signatures, on the other hand, and especially that of 
the fluorescent line, is generally subject to significantly less variations. Therefore they can be easily identified in the frequency resolved spectra and their amplitude can be measured. Absence or presence of the reflection signatures in a Fourier frequency resolved spectrum would signal absence or presence of variations of the reflected emission at the given frequency. Their amplitude would in principal measure amplitude of the variations of the reflected flux relative to the variations of the primary emission. With that in mind we show in Fig. 4 the Fourier frequency resolved spectra of GX 339-4 in two frequency ranges. Significant decrease of the amplitude of the reflection features with frequency is apparent. Quantitative dependence of the reflection amplitude on the Fourier frequency is shown in Fig. 5. Within the available statistical accuracy this dependence is qualitatively and quantitatively similar to that found for Cyg X-1 (Revnivtsev et al. 1999).

\section{Discussion}

We analyzed 23 observations of GX 339-4 with RXTE/ PCA performed from 1996-1997 during the low spectral state of the source. Using simple spectral model we found that the pattern of temporal and spectral variability of GX 339-4 is confirming the previous findings of Ueda et al. (1994), obtained with the help of GINGA observatory, and it is nearly identical to that of Cyg X-1 (Revnivtsev et al. 1999, Paper I). This indicates that such a pattern might be common for the accreting black holes in the low spectral state. In particular:

1. The characteristic noise frequency, slope of the Comptonized spectrum and amplitude of the reflected component change in a correlated way. Increase of the noise frequency is accompanied by increase of the amplitude of the reflected component and steepening of the Comptonized spectrum.

2. Fourier frequency resolved spectral analysis showed that the short term variations of the reflected flux are suppressed in comparison with variations of the primary Comptonized flux at frequencies above $\sim 1-10 \mathrm{~Hz}$.

As it was discussed e.g. in Gilfanov et al. (1999), Zdziarski et al. (1999) the correlation of the spectral index (the photon index $\Gamma$ of the underlying power law) with the amplitude of the reflected component hints on the possible relation between the solid angle, subtended by the reflector, and the influx of the soft photons to the Comptonization region. Existence of such relation suggests that the reflecting medium is the primary source of the soft seed photons to the Comptonizing region. It could be explained e.g. within the framework of the disk-spheroid models of the accretion flow (see e.g. Poutanen et al. 1997) (a hot quasispherical Comptonization region near the compact object surrounded by an optically thick cold accretion disk terminating at some radius $R_{\text {in }}$ ). In such geometry the decrease of the inner radius of the optically thick cold accretion disk should result in: 1) the decrease of the temperature of the inner hot region, because of the increase of the influx of cold seed photons, and 2) the increase of the solid angle subtended by this accretion disk (reflector) seen from the central hard source. This would lead to the correlation of the spectral index and the amplitude of the reflection. In addition, if we assume that the characteristic frequencies of aperiodic variations of the source flux are proportional to the keplerian frequency on the inner boundary of the optically thick accretion disk we will obtain an additional correlation. The characteristic frequency of the source's power spectrum will positively correlate with the amplitude of the reflection. This is what we observe in the cases of Cyg X-1 and GX 339-4. We should note, however, that the above explanation of the observed $R-\Gamma$ correlation is not unique. For example, the similar dependencies can be produced in the model of active corona above the accretion disk (see e.g. Beloborodov 1999; Gilfanov et al. 2000a).

The frequency resolved spectral analysis was introduced in Revnivtsev et al. (1999). In that paper we showed that the reflected component in the spectrum of Cyg X-1 is less variable than the underlying continuum at the frequencies above $\sim 1 \mathrm{~Hz}$. The similar behavior, but with less statistical significance was found in the case of GX 3394. Similar to the discussion in Revnivtsev et al. (1999) we can assume here that the time variations of the reflected component in the spectrum of GX 339-4 could be smeared out by the finite light-crossing time of the reflector (see more extended discussion in Gilfanov et al. 2000b). Alternatively, the observed behavior could be explained by non-uniformity within the comptonizing region. For example, if the short time scale variations appear in geometrically inner part of the accretion flow (hot spheroid) and give a rise to significantly weaker, if any, reflected emission than the longer time scale events (originating in the outer regions) then we will see no reflection features at high Fourier frequencies. In turn, the smaller amplitude of the reflection in the inner regions of the accretion flow might be caused by the screening of the reflector from the innermost regions by the outer parts of spheroid.

Acknowledgements. This research has made use of data obtained through the High Energy Astrophysics Science Archive Research Center Online Service, provided by the NASA/Goddard Space Flight Center. MR acknowledges partial support from RBRF grant 97-02-16264.

\section{References}

Beloborodov, A. 1999, ApJ, 510, L123

Fabian, A., Rees, M., Stella, L., \& White, N. 1989, MNRAS, 238,729

Gilfanov, M., Churazov, E., \& Revnivtsev, M. 1999, A\&A, 352, 182 
Gilfanov, M., Churazov, E., \& Revnivtsev, E. 2000a, Proc. of 5th Sino-German Workshop on Astrophysics SGSC Conf. Ser., 1 (China Science \& Technology Press Beijing), ed. G. Zhao, J. J. Wang, H. M. Qiu, G. Boerner, 114 [astro-ph/0002415]

Gilfanov, M., Churazov, E., \& Revnivtsev, M. 2000b, MNRAS, 316,923

Jahoda, K. 1999 http://Iheawww.gsfc.nasa.gov/users/ keith/pcarmf.html

Magdziarz, P, \& Zdziarski, A. 1995, MNRAS, 273, 837

Nowak, M., Wilms, J., \& Dove, J. 1999, ApJ, 517, 335

Poutanen, J., Krolik, J., \& Ryde, F. 1997, MNRAS, 292, L21

Revnivtsev, M., Gilfanov, M., \& Churazov, E. 1999, A\&A, 347, L23
Stark, M. 1999 http://lheawww.gsfc.nasa.gov/ stark/pca/ pcabackest.html

Tanaka, Y., \& Lewin, W. H. G. 1995, in X-ray Binaries, ed. W. H. G. Lewin, J. van Paradijs, \& E. P. J. van der Heuvel (Cambridge Univ. Press, Cambridge), p. 126

Trudolyubov, S., Gilfanov, M., Churazov, E., et al. 1998, A\&A, 334,895

Ueda, Y., Ebisawa, K., \& Done, C. 1994, PASJ, 46, 107

Wilms, J., Nowak, M., Dove, J., Fender, R., \& Di Matteo, T. 1999, ApJ, 522, 460

Zdziarski, A. A., Poutanen, Ju., Mikolajewska, J., et al. 1998, MNRAS, 301, 435

Zdziarski, A. A., Lubinski, P., \& Smith, D. 1999, MNRAS, 303, L11 